\title{
Mesenchymal stromal cells of osteosarcoma patients do not show evidence of neoplastic changes during long-term culture
}

\author{
Emilie P Buddingh', S Eriaty N Ruslan', Christianne M A Reijnders², Karoly Szuhai ${ }^{3}$, Marieke L Kuijjer², \\ Helene Roelofs ${ }^{4}$, Pancras C W Hogendoorn², R Maarten Egeler ${ }^{1}$, Anne-Marie Cleton-Jansen ${ }^{2+}$ \\ and Arjan C Lankester ${ }^{1 *+}$
}

\begin{abstract}
Background: In vitro expanded mesenchymal stromal cells (MSCs) are increasingly used as experimental cellular therapy. However, there have been concerns regarding the safety of their use, particularly with regard to possible oncogenic transformation. MSCs are the hypothesized precursor cells of high-grade osteosarcoma, a tumor with often complex karyotypes occurring mainly in adolescents and young adults.

Methods: To determine if MSCs from osteosarcoma patients could be predisposed to malignant transformation we cultured MSCs of nine osteosarcoma patients and five healthy donors for an average of 649 days (range $601-$ 679 days). Also, we compared MSCs derived from osteosarcoma patients at diagnosis and from healthy donors using genome wide gene expression profiling.

Results: Upon increasing passage, increasing frequencies of binucleate cells were detected, but no increase in proliferation suggestive of malignant transformation occurred in MSCs from either patients or donors. Hematopoietic cell specific Lyn substrate 1 (HLCS1) was differentially expressed (fold change $0.25, P$ value 0.0005 ) between MSCs of osteosarcoma patients $(n=14)$ and healthy donors $(n=9)$.

Conclusions: This study shows that although HCLS1 expression was downregulated in MSCs of osteosarcoma patients and binucleate cells were present in both patient and donor derived MSCs, there was no evidence of neoplastic changes to occur during long-term culture.
\end{abstract}

\section{Background}

Mesenchymal stromal cells (MSCs) are increasingly used as experimental cellular therapy in a wide range of conditions, such as graft-versus-host disease in the context of allogeneic bone marrow transplantation, auto-immune diseases and for regenerative purposes in for example myocardial injury or cartilage defects [1-6]. However, since MSCs have to be expanded in vitro to achieve sufficient numbers, there have been concerns regarding the

\footnotetext{
*Correspondence: a.lankester@lumc.nl

${ }^{\dagger}$ Anne-Marie Cleton-Jansen and Arjan C Lankester contributed equally to this work

1 Department of Pediatrics, J6-S, Leiden University Medical Center, PO Box 9600, 2300 RC Leiden, The Netherlands

Full list of author information is available at the end of the article
}

safety of their use, particularly with regard to possible oncogenic transformation [7]. Cultured murine MSCs readily transform and form sarcoma-like tumors in vivo [8-12]. Similarly, MSCs derived from rhesus macaques become polyploid and subsequently aneuploid during long-term culture [13]. In contrast, human MSCs appear resistant to spontaneous in vitro transformation [14]. Studies reporting that human MSCs undergo malignant transformation in vitro have been retracted because of cross-contamination issues [15-20]. Despite the apparent difference between human and murine MSCs in their propensity to spontaneously transform in vitro, concerns remain. MSCs are hypothesized to be the precursor cells of high-grade osteosarcoma (OS) and a patient transplanted with bone marrow (containing hematopoietic 
stem cells and MSCs) from a sibling was diagnosed with OS originating from donor stem cells 17 years later [21]. This case demonstrates that donor-derived (pre-) cancerous MSCs can survive in a host and cause disease many years later. Another cause for concern is the observation that cultured MSCs can acquire chromosomal aberrations, although these do not seem to confer a selective growth advantage in vitro $[22,23]$.

High-grade osteosarcoma is a malignant primary bone tumor which often occurs at a relatively young age [24]. OS tumor cells are characterized by aneuploid karyotypes and gross chromosomal instability [25]. Such highly complex chromosomal rearrangements can occur as a result of a single catastrophic event, termed chromothripsis $[26,27]$. However, this probably has to occur in a susceptible background, either as a genetic predisposition or acquired as a de novo event. In a murine model failed cytokinesis can lead to tetraploidy and subsequent tumorigenesis only in a p53 deficient host [28]. We previously showed loss of CDKN2A/p16 protein expression in tetraploid tumorigenic murine MSCs [9]. We hypothesized that normal MSCs from OS patients could be predisposed to malignant transformation, and performed long term in vitro culture and genome wide expression profiling of early passage MSCs from OS patients and healthy donors. Here we show that OS patient-derived MSCs do not transform in vitro, confirming previous reports in healthy individuals that spontaneous transformation of human MSCs in vitro is an extremely unlikely event.

\section{Methods}

\section{Patients}

Characteristics of OS patients and healthy stem cell donors can be found in Table 1. Bone marrow cells of OS patients were harvested under general anesthesia prior to start of the chemotherapeutic treatment. The site of MSC harvest (iliac crest) was different from the location of the primary tumor (metaphyseal ends of the long bones) in all cases. Healthy donors were either identical sibling donors for patients with malignant or benign disease requiring hematopoietic stem cell transplantation; or haploidentical donors for either hematopoietical stem

Table 1 Characteristics of osteosarcoma patients (OS) and healthy donors (HD) and overview of experiments

\begin{tabular}{|c|c|c|c|c|c|c|c|c|}
\hline \multirow[t]{2}{*}{ Culture } & \multirow[t]{2}{*}{ OS (UI)/HD } & \multirow[t]{2}{*}{ Sex } & \multirow[t]{2}{*}{ Age (years) } & \multirow[t]{2}{*}{ Histology } & \multirow[t]{2}{*}{ Location of the primary tumor } & \multicolumn{3}{|c|}{ Included in experiment } \\
\hline & & & & & & Microarray & qPCR & Long-term culture \\
\hline $0010 S$ & OS (352) & M & 12 & Osteoblastic & Distal femur & Y & Y & Y \\
\hline $0020 S$ & OS (340) & $\mathrm{F}$ & 13 & Osteoblastic & Distal femur & Y & Y & Y \\
\hline o03OS & OS (376) & $\mathrm{F}$ & 13 & Telangiectatic & Distal femur & Y & Y & Y \\
\hline $0040 S$ & OS (377) & $\mathrm{F}$ & 14 & Sclerosing & Proximal tibia & Y & Y & Y \\
\hline $0050 s$ & OS (348) & $\mathrm{F}$ & 15 & Telangiectatic & Distal tibia & $N$ & $\mathrm{~N}$ & Y \\
\hline 00605 & OS (349) & M & 8 & Osteoblastic & Distal femur & Y & Y & Y \\
\hline 0070s & OS (350) & $\mathrm{F}$ & 15 & Osteoblastic & Distal femur & $\mathrm{N}$ & $\mathrm{N}$ & Y \\
\hline 0080s & OS (378) & $\mathrm{F}$ & 9 & Osteoblastic & Proximal humerus & Y & Y & Y \\
\hline 0090s & OS (382) & M & 15 & Chondroblastic & Proximal humerus & Y & Y & Y \\
\hline $0100 \mathrm{~s}$ & OS (388) & $M$ & 14 & Osteoblastic & Proximal humerus & $N$ & Y & $N$ \\
\hline $0110 \mathrm{~s}$ & OS (391) & $\mathrm{F}$ & 14 & Osteoblastic & Proximal tibia & $N$ & Y & $N$ \\
\hline $0120 \mathrm{~S}$ & OS (394) & $\mathrm{F}$ & 5 & Osteoblastic & Distal femur & $\mathrm{N}$ & Y & $\mathrm{N}$ \\
\hline 01305 & OS (393) & F & 14 & Osteoblastic & Distal femur & $N$ & Y & $N$ \\
\hline $0140 S$ & OS (395) & $M$ & 10 & Sclerosing & Distal femur & $\mathrm{N}$ & Y & $\mathrm{N}$ \\
\hline $0150 \mathrm{~S}$ & OS (396) & $M$ & 13 & Osteoblastic & Distal femur & $\mathrm{N}$ & Y & $\mathrm{N}$ \\
\hline $0160 \mathrm{~S}$ & OS (402) & $\mathrm{F}$ & 15 & Sclerosing & Proximal tibia & $N$ & Y & $\mathrm{N}$ \\
\hline $\mathrm{HB}$ & $\mathrm{HD}(\mathrm{HB})$ & $M$ & 15 & & & Y & Y & Y \\
\hline HD3 & $\mathrm{HD}(\mathrm{HD} 3)$ & $\mathrm{F}$ & 27 & & & Y & Y & $Y$ \\
\hline HD5 & $\mathrm{HD}(\mathrm{HD} 5)$ & $M$ & 50 & & & Y & Y & Y \\
\hline $\mathrm{MH}$ & $\mathrm{HD}(\mathrm{MH})$ & $M$ & 15 & & & Y & Y & Y \\
\hline TD1 & HD (TD1) & $\mathrm{F}$ & 11 & & & Y & Y & Y \\
\hline TD2 & HD (TD2) & $\mathrm{F}$ & 43 & & & $\mathrm{~N}$ & Y & $\mathrm{N}$ \\
\hline TD3 & $\mathrm{HD}(\mathrm{TD} 3)$ & $\mathrm{F}$ & 43 & & & $\mathrm{~N}$ & Y & $\mathrm{N}$ \\
\hline TD4 & HD (TD4) & $\mathrm{F}$ & 25 & & & $\mathrm{~N}$ & Y & $\mathrm{N}$ \\
\hline TD5 & HD (TD5) & $M$ & 5 & & & $N$ & $Y$ & $N$ \\
\hline
\end{tabular}

Ul unique identifier. 
cell transplantation or the therapeutic infusion of MSCs for steroid-resistant graft-versus-host disease. Written informed consent was obtained from all patients and donors prior to bone marrow harvesting. The study was approved by the Institutional Review Board on Medical Ethics [LUMC Medical Ethics Committee (CME), P06.152].

\section{Mesenchymal stromal cell cultures}

Bone marrow derived mononuclear cells were obtained from 5 to $15 \mathrm{~mL}$ of heparinized bone marrow aspirate by density gradient centrifugation on Ficoll. Cells were plated on non-coated $75 \mathrm{~cm}^{2}$ polystyrene flasks at a cell density of $160,000 / \mathrm{cm}^{2}$ in complete culture medium (LG-DMEM; Invitrogen, Paisley, UK) supplemented with penicillin/streptomycin (Invitrogen) and 10\% fetal bovine serum (FBS; HyClone, Verviers, Belgium). We used a characterized and defined FBS batch preselected for its potential to support MSC expansion and continued to use this specific batch throughout the culture period. MSCs were plastic adherent and had spindle shaped morphology. Chondrogenic, adipogenic and osteoblastic differentiation were performed as described earlier [29]. Medium was refreshed twice a week and cells were replated when reaching $80-90 \%$ confluence at a density of $4,000 / \mathrm{cm}^{2}$. The first nine OS patient and first five healthy donor MSC samples that were obtained were cultured long-term, the subsequent samples were used for confirmatory mRNA expression analysis (Table 1 ). Morphology of the cells was recorded and population doublings per passage (PD) were calculated using the $\log$ ratio of the harvesting cell count $(\mathrm{N})$ to the starting (baseline) count (X0), divided by the $\log$ of $2(\mathrm{PD}=[\log$ $(\mathrm{N} / \mathrm{X} 0)] / \log 2)$.

\section{Flow cytometry}

Expression of the cell surface markers CD73, CD90 and CD105 and absence of hematopoietic markers on MSCs was determined using flow cytometry, according to the statement by the International Society for Cellular Therapy [30]. Cells were detached using Trypsin/EDTA and washed in PBS/0.05\% bovine serum albumin. Antibodies used were (FITC) conjugated anti-CD86-fluorescein isothiocyanate (FITC) (cat. no. 555657), anti-HLA-DR-FITC (cat. no. 347400), anti-CD31-phycoerythrin (PE) (cat no 555446), anti-CD34-PE (cat no 348057), anti-CD73PE (cat no 550257), anti-CD90-PE (cat no 555596), anti-CD3-peridinin chlorophyll protein(PerCP)-Cy5.5 (cat no 332771), anti-CD45-PerCPCy5.5 (cat no 332784), all from BD (San Diego, CA, USA) and anti-CD105-PE (cat no SN6), from Ancell (Bayport, MN, USA). Flow cytometry was performed on a FACScalibur, and analyzed using Cellquest software (both Becton-Dickinson).
Mean fluorescence intensity (MFI) ratio was calculated by determining the MFI of the specific staining relative to the MFI of the appropriate isotype control staining.

\section{Analysis of binucleate cells}

MSCs were grown on coverslips. Coverslips were washed with PBS and fixed in 4\% PFA. Coverslips were incubated for $10 \mathrm{~min}$ in $1 \mu \mathrm{g} / \mathrm{mL}$ acridine orange (Sigma-Aldrich, Zwijndrecht, the Netherlands) to stain cytoplasm and $1 \mu \mathrm{g} / \mathrm{mL}$ wheat-germ agglutinin with an Alexa Fluor 594 conjugate (Invitrogen) in PBS to stain cell membranes. Following washes in PBS, coverslips were mounted using Vectashield with DAPI (Vector laboratories, Burlingame, CA, USA) to stain nuclei. Images were acquired using a COHU 4910 series monochrome CCD camera (COHU, San Diego, CA, USA) attached to a DM fluorescence microscope (Leica, Wetzlar, Germany) and analyzed using Image software with the Cell Counter plug-in (National Institute of Mental Health, Bethesda, MD, USA).

\section{RNA isolation}

RNA was isolated from frozen cell pellets of at least $1^{*} 10^{\mathrm{e}} 6$ undifferentiated MSCs at passage 2-5 using TRIzol reagent (Invitrogen). Cells were lysed in TRIzol, followed by phase separation in chloroform, precipitation using 2-propanolol and washing in 75\% ethanol. RNA clean-up was performed using the QIAGEN Rneasy mini kit (Venlo, the Netherlands) with on-column DNAse treatment. RNA quality and concentration were measured using an Agilent 2100 Bioanalyzer (Santa Clara, CA, USA) and Nanodrop ND-1000 (Thermo Fisher Scientific, Waltham, MA, USA), respectively.

\section{CDNA synthesis, cRNA amplification, and Illumina Human v2.0 Expression BeadChip hybridization}

Gene expression profiling was performed using Human-6 v2 Expression BeadChips (San Diego, CA, USA) containing $>48,000$ transcript probes. Synthesis of cDNA, cRNA amplification, and hybridization of cRNA onto the Illumina Human v2.0 Expression BeadChips were performed as described previously [31, 32].

\section{qPCR}

cDNA synthesis for qPCR was performed as described earlier [32]. qPCR was performed using the $\mathrm{iQ}^{\mathrm{m}} \mathrm{SYBR}^{\circ}$ Green Supermix (Bio-Rad, Hercules, CA, USA) according to the manufacture's instructions, reverse transcriptase negative (RT-) and $\mathrm{H}_{2} \mathrm{O}$ controls were taken along for each sample. For normalization, three genes with stable expression in the microarray experiments were chosen (CPSF6, GPR108 and CAPNS1). Data were normalized by geometric mean expression levels of the 
three reference genes using geNorm (http://medgen. ugent.be/jvdesomp/genorm/). Primer sequences can be found in Additional file 1: Table S1. A standard curve was taken along for each primer set and used for quantification, PCR efficiencies ranged from 93 to $104 \%$.

\section{Karyotyping}

Six early passage samples (three derived from OS patients, three derived from healthy donors) were subjected to a multicolor FISH based karyotyping test (COBRA-FISH) as described earlier [33]. From each sample at least 20 metaphase cells were recorded and analyzed.

\section{Statistical analysis}

Microarray data were normalized using the Cubic Spline normalization method with the Illumina BeadStudio Gene Expression Module. Microarray data are available at GEO using the accession no. GSE42572. Statistical analysis of microarray was performed using Significance Analysis for Microarrays, using a false discovery rate of 20\% (SAM, http://www-stat.stanford.edu/ tibs/SAM/) [34]. Univariate statistical analyses were performed using GraphPad Prism software (version 5.01, La Jolla, CA, USA). Two-sided $P$ values lower than 0.05 were determined to be significant.

\section{Results}

MSCs of OS patients and healthy donors do not differ in expression of cell surface markers or differentiation capacity

We tested the MSC cultures from OS patients and healthy donors for phenotypic markers and functionality. Samples from 16/16 patients and 9/9 controls were able to differentiate into chondrogenic (Additional file 2: Figure S1A), adipogenic (Additional file 2: Figures S1B and $\mathrm{C}$ ) and osteoblastic lineages (Additional file 2: Figures S1D and E). Also, all MSC cultures expressed CD73, CD90 and CD105 and lacked expression of hematopoietic markers (Additional file 2: Figure S1F). Level of expression of CD73, CD90 and CD105 as determined by MFI-ratios (specific staining/isotype control) did not differ between MSC cultures derived from OS patients and healthy donors. Early passage samples from three OS patient derived MSCs and three healthy donor derived MSCs were karyotyped; no structural or numerical aberrations were observed in the analyzed samples (Additional file 3: Figure S2): MSC001: 46,XY (26 metaphases), MSC003: 46,XX (27 metaphases), MSC008:46,XX (22 metaphases), MSC-HB: 46,XY (21 metaphases), MSCMH: 46,XY (20 metaphases), MSC-TD: 46,XX (21 metaphases). Late passage cells could not be karyotypically analyzed due to the low numbers of dividing cells.

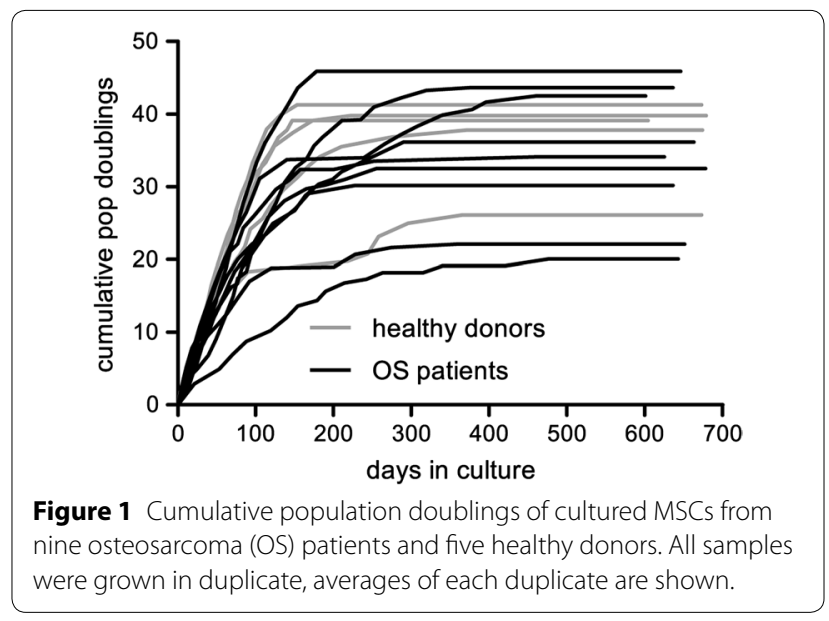

Long-term in vitro culture of MSCs results in increased binucleation but not in malignant transformation

MSCs of nine OS patients and five healthy donors were long term cultured (average number of days in culture 649, range 601-679 days). From each individual sample, duplicate cultures were established. There were no significant differences between growth rate, cumulative population doublings (median cumulative population doublings OS patients 34 vs. healthy donors 39; $P$ value Mann-Whitney $U$ test 0.70 ), passage number at termination of culture (mean passage number OS patients 21 vs. healthy donors 23; $P$ value Mann-Whitney $U$ test 0.74 ) or time to growth arrest (median days to growth arrest OS patients 441 days vs. healthy donors 222 days; $P$ value Mann-Whitney U test 0.15 ) between MSCs of OS patients and healthy donors. The cumulative population doublings are shown in Figure 1 (averages of the duplicate cultures are shown per sample). All cultures exhibited rapid exponential growth in the first few passages. Later, proliferation slowed down and eventually stopped, characteristic of cultured cells in crisis. At termination of the cultures, viable cells were present in all samples. Cultures were terminated at the end of the growth curves. At this point, in none of the cultures there was evidence of cells escaping the crisis by an increase in proliferation and corresponding morphological changes indicative for spontaneous in vitro malignant transformation.

Morphology of the cells was inspected with every passage. Low passage cells were spindle-shaped, higher passage cells were larger. Upon increasing passage number, increasing frequencies of binucleate cells were noted using phase contrast microscopy. To quantify binucleation, cells were grown on coverslips and cytoplasm, cell membranes and nuclei were stained. Mono- and binucleate cells were counted (Figure $2 \mathrm{a}-\mathrm{d}$ ). There was no difference in percentage of binucleate cells between MSCs derived from OS patients and healthy donors (Figure 2e). 


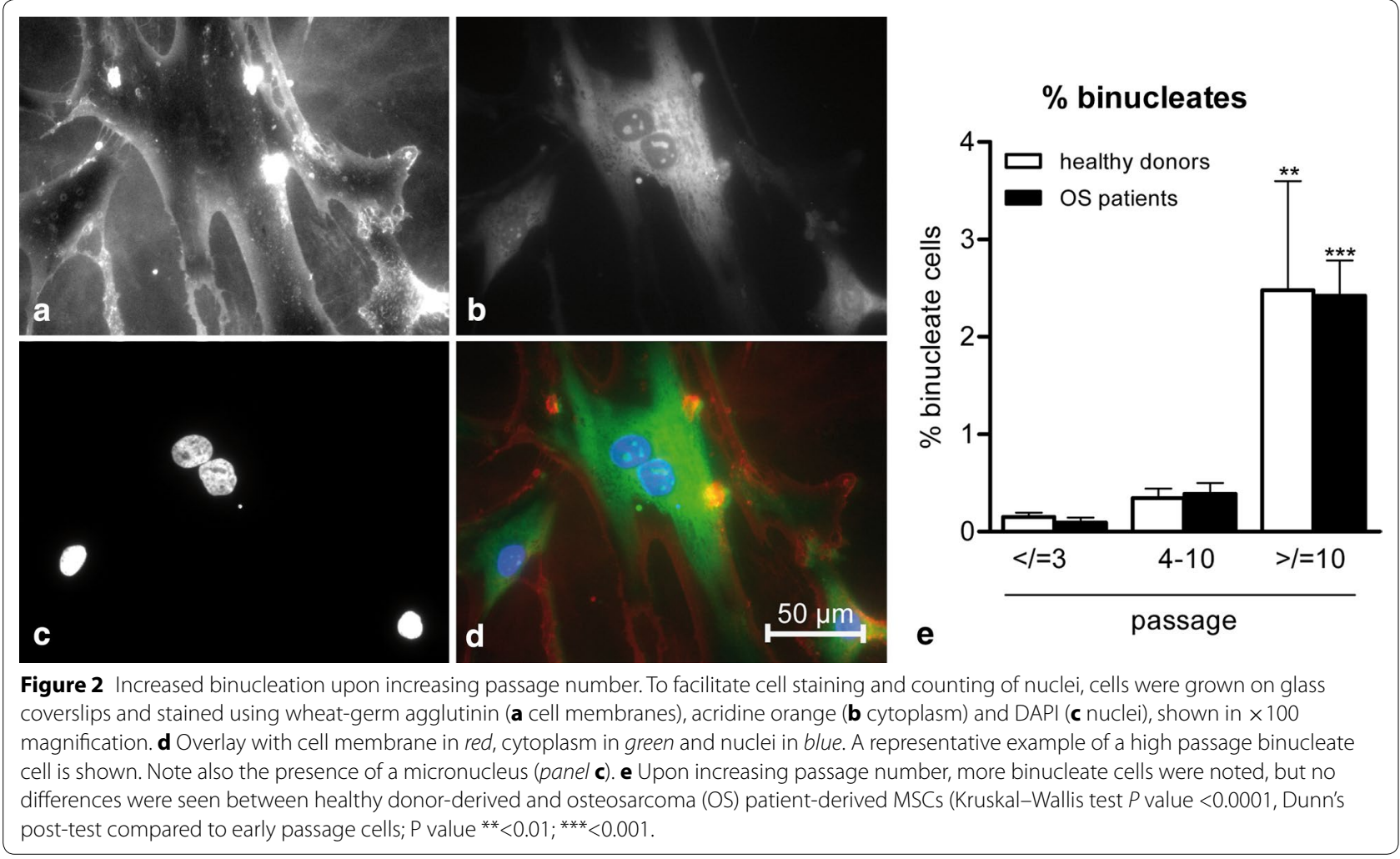

\section{HCLS1 mRNA expression is downregulated in OS patient derived MSCs}

Using microarray gene expression analysis, five genes were found to be differentially expressed between early passage healthy donor derived MSCs $(n=5)$ and OS patient derived MSCs $(\mathrm{n}=7)$, with a false discovery rate of $20 \%$. Expression of HCLS1, ADM, EEF1A1, LOC644739 (or WASF4) and LOC441155 was lower in patient derived MSCs as compared to healthy donor derived MSCs. To perform simultaneous technical and biological validation of the microarray results, the original low passage MSC samples were recultured and the original series expanded to now include four additional healthy donor derived MSCs (total of $n=9$ ) and seven additional OS patient derived MSCs (total of $n=14$ ). Four of the differentially expressed genes were validated using qPCR: hematopoietic cell specific Lyn substrate 1 (HCLS1), adrenomedullin $(A D M)$, eukaryotic translation elongation factor 1 alpha 1 (EEF1A1) and LOC644739 (or WAS protein family, member 4; WASF4). LOC441155 is a pseudogene for which we did not succeed in designing a sufficiently specific and efficient primer pair. In this second, newly cultured and expanded series, expression of HCLS1 was significantly lower in OS patient derived MSCs as compared to healthy donor derived MSCs (Figure 3 , fold change $0.25, P$ value 0.0005 ). The other genes were not differentially expressed in this series, perhaps because this was a newly expanded batch of cells. We tried to determine if the observed difference in HCLS1 expression was also true at the protein level. Although HCLS1 protein could be clearly detected in the positive control cell line Jurkat, we were unable to reproducibly detect HCLS1 in MSCs. For five OS patients we had mRNA available from both bone-marrow derived MSCs and the primary tumor. In these samples, expression of HCLS1 was higher in tumor tissue as compared to MSCs, but there was no correlation between level of expression of HCLS1 in low passage MSCs and in the corresponding tumor tissue.

\section{Discussion}

In recent years, MSCs have gained increasing interest as a therapeutic modality for immune modulatory and regenerative purposes [1-4]. In order to achieve sufficient numbers of cells for therapeutic utility, MSCs need to be expanded in vitro prior to infusion. Since long-term culture of non-human MSCs can result in oncogenic transformation and the occurrence of sarcomas in the receiving hosts, there is ongoing debate regarding the safety of using cultured MSCs in clinical studies [35]. In the present study, we compared human MSCs derived from OS patients at diagnosis and from healthy donors 


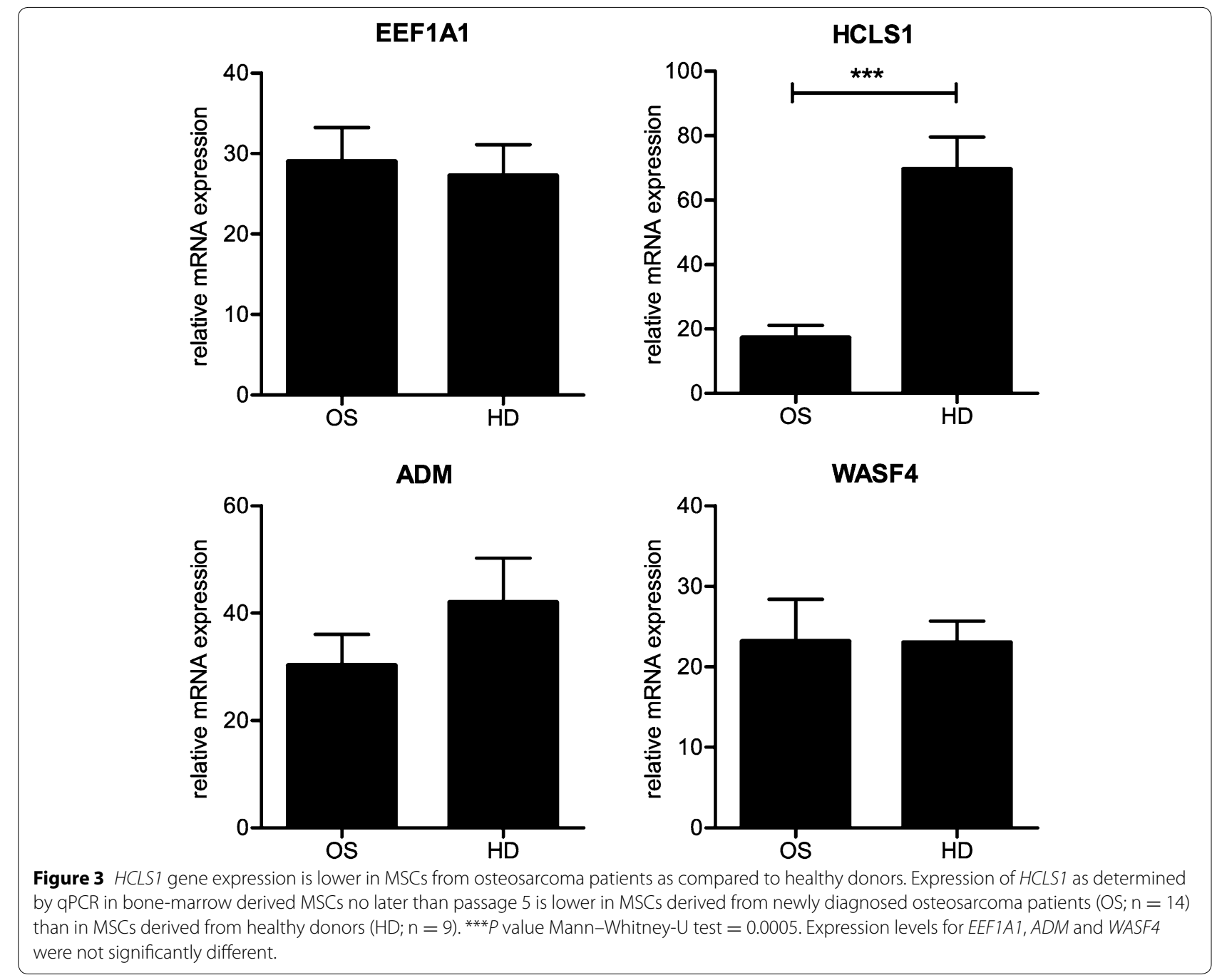

to answer two questions. First, do human MSCs transform to a malignant phenotype when cultured in vitro, as has been shown for murine MSCs? Second, are MSCs derived from OS patients more likely to transform than healthy donor derived MSCs?

MSCs from patients and controls exhibited similar growth patterns during long-term in vitro culture. During this time, all cultures reached a plateau in proliferation characteristic of cells in crisis. In contrast to what we have previously observed in murine MSCs [9], we never observed escape of this crisis by a rapidly dividing cell population with changed morphology, even after almost 2 years of continuous culture. There were no phenotypical or functional differences between OS patient derived and healthy donor derived MSCs and early passage karyotypes were normal in both groups. Differentiation capacity and expression of specific cell surface markers was similar.

During prolonged culture, progressive shortening of telomeres occurs [36]. This can cause anaphase chromatin bridges, resulting in failed cytokinesis and consequently in binucleate cells [37]. In the context of loss of expression or function of tumor suppressor proteins and corresponding cell cycle checkpoints, tetraploidy and ultimately aneuploidy will occur [28]. Upon increasing passage, increasing frequencies of binucleate cells were noted; both in patient derived MSCs and healthy donor derived MSCs (Figure 2). However, since we did not observe an increase in proliferation in these cells despite the presence of increasingly high frequencies of binucleate cells, cell cycle checkpoints were probably functionally intact, both in MSCs derived from OS patients and from healthy donors.

In addition to the functional read-out of long-term culture, we also performed gene expression analysis on early passage cells. Expression of HCLS1 was downregulated in MSCs from OS patients as compared to MSCs of healthy donors. HCLS1 is primarily known for its role in the signaling cascade that follows $\mathrm{B}$ cell receptor activation. It is 
highly expressed in B-cell derived malignancies and is associated with a poor outcome [38, 39]. Further studies are needed to determine if HCLS1 might have a tumor suppressor function and if its loss of expression in OS patients derived MSCs has any relationship to in vivo tumorigenesis. Although we show no functional difference between healthy donor and OS patient derived MSCs, we do not advocate the use of MSCs from known cancer patients for clinical purposes. The relevance of the differential expression of HCLS1 remains unknown and there might be other undetected (pre-) malignant alterations.

Both on a transcriptional and on a functional level, OS patient and healthy donor derived MSCs were very similar. There are several possible explanations for the observed similarities between OS patient derived and donor derived MSCs. First, we obtained MSCs from the iliac crest, while all tumors in our series were located at the metaphyseal ends of the long bones. OS occurs at a time and place of active growth and perhaps this proproliferative microenvironment (with high expression of growth factors) is an essential prerequisite for oncogenic transformation of MSCs. Second, (pre-) oncogenic alterations may be present in only one or a few local mesenchymal tumor precursor cells and not in MSCs at a distant site such as the iliac crest. This could be due to somatic mosaicism, similar to what has been shown for the enchondromatosis syndromes Ollier disease and Maffucci syndrome [40]. Third, according to the 'multiple hit hypothesis', there may not have been sufficient 'hits' for in vitro transformation to occur, even if relevant predisposing alterations are present. Ionizing radiation is a well-known risk factor for developing osteosarcoma and low-dose radiation facilitates oncogenic transformation in telomerase-transduced immortalized MSCs [41, 42]. Perhaps one or two additional 'hits' (for example loss of cell cycle checkpoint control or radiation-induced DNA damage) would be enough for oncogenic transformation to occur.

Fourth, pre-malignant alterations may have been present in only a few cells, which would not lead to large enough differences in gene expression to be picked up by genome wide expression analysis. Novel techniques to study genetic alterations at a single cell level might be able to pick up these rare events [43,44]. Finally, although there is compelling evidence to suggest MSCs are the precursor cells for human OS, this has not yet been unequivocally proven.

\section{Conclusions}

During long-term in vitro culture of human OS patient and healthy donor derived MSCs, there was no evidence for neoplastic changes to occur. We could not confirm our hypothesis that MSCs of OS patients might have a higher propensity to oncogenic transformation than healthy donor derived MSCs. In contrast to what has been reported for other species, under the tested conditions, human MSCs do not easily transform. Although we cannot exclude the occurrence of low frequencies of cells with genomic alterations, we did not see a selective growth advantage of aberrant cells nor did we observe karyotypic abnormalities in low passage cells. This data supports the currently held view that administration of low passage cultured healthy donor derived human MSCs for therapeutic purposes is unlikely to result in sarcomas in the host.

\section{Additional files}

Additional file 1: Table S1. Primer sequences used for qPCR experiments.

Additional file 2: Figure S1. Differentiation capacity and expression of membranous markers of MSCs. Representative examples are shown (40x magnification). A, chondrogenic differentiation was performed using cell pellet cultures and assessed using toluidine blue staining. Adipogenic differentiation was assessed using phase contrast microscopy (B) and Oil-red-O staining (C). Osteogenic differentiation was assessed using phase contrast microscopy (D) and Alizarin red staining (E). F, all samples expressed CD73, CD90 and CD105 (bold histograms) as determined by flow cytometry (isotype controle staining shown for comparison).

Additional file 3: Figure S2. Representative example of karyotypic analysis of early passage MSCs (OS patient derived MSC003OS; 46,XX).

\section{Abbreviations}

ADM: adrenomedullin; EEF1A1: eukaryotic translation elongation factor 1 alpha 1; FBS: fetal bovine serum; FITC: fluorescein isothiocyanate; HCLS1: hematopoietic cell specific lyn substrate 1; MFI: mean fluorescence intensity; MSC: mesenchymal stromal cell; OS: osteosarcoma; PD: population doublings; PE: phycoerythrin; PerCP: peridinin chlorophyll protein; WASF4: WAS protein family member 4.

\section{Authors' contributions}

EB participated in design of the study, harvested and cultured bone marrow derived MSCs and performed and analyzed flow cytometry, differentiation assays, binucleate cell assays and GPCR, and drafted the manuscript. SR cultured bone marrow derived MSCS and performed and analyzed flow cytometry, differentiation assays, binucleate cell assays and gPCR. CR cultured bone marrow derived MSCs and performed and analyzed flow cytometry and differentiation assays. KS performed and analyzed karyotypic analyses. MK performed and analyzed genome wide profiling studies. HR harvested, cultured and analyzed BM derived MSCs. PH and RE participated in design of the study and helped to draft the manuscript. AC and AL conceived of the study, participated in the design and coordination and helped to draft the manuscript. All authors read and approved the final manuscript.

\section{Author details}

1 Department of Pediatrics, J6-S, Leiden University Medical Center, PO Box 9600, 2300 RC Leiden, The Netherlands. ${ }^{2}$ Department of Pathology, Leiden University Medical Center, Leiden, The Netherlands. ${ }^{3}$ Department of Molecular Cell Biology, Leiden University Medical Center, Leiden, The Netherlands.

${ }^{4}$ Department of Immunohematology and Blood Transfusion, Leiden University Medical Center, Leiden, The Netherlands.

\section{Acknowledgements}

The authors like to thank Ronald Duim for technical assistance, Marco Schilham for critical review, and Maarten Morsink for help in analysis of microarray 
data. Also, the authors would like to thank the medical and nursing staff of the departments of pediatrics and clinical oncology for help in obtaining informed consent. This work was financially supported by EuroBoNet, a European Commission granted Network of Excellence for studying the pathology and genetics of bone tumors (Grant Number LSHC-CT-2006-018814) and by the Netherlands Organization for Health Research and Development (ZonMw, Grant Number 92003-399 to EB).

\section{Compliance with ethical guidelines}

\section{Competing interests}

The authors declare that they have no competing interests.

Received: 17 April 2015 Accepted: 12 June 2015

Published online: 23 June 2015

\section{References}

1. Le Blanc K, Frassoni F, Ball L, Locatelli F, Roelofs H, Lewis I et al (2008) Mesenchymal stem cells for treatment of steroid-resistant, severe, acute graft-versus-host disease: a phase II study. Lancet 371:1579-1586

2. Uccelli A, Prockop DJ (2010) Why should mesenchymal stem cells (MSCs) cure autoimmune diseases? Curr Opin Immunol 22:768-774

3. Orlic D, Kajstura J, Chimenti S, Jakoniuk I, Anderson SM, Li B et al (2001) Bone marrow cells regenerate infarcted myocardium. Nature 410:701-705

4. Cipriani P, Carubbi F, Liakouli V, Marrelli A, Perricone C, Perricone R et al (2013) Stem cells in autoimmune diseases: implications for pathogenesis and future trends in therapy. Autoimmun Rev 12(7):709-716

5. Ball LM, Bernardo ME, Roelofs H, Lankester A, Cometa A, Egeler RM et al (2007) Cotransplantation of ex vivo-expanded mesenchymal stem cells accelerates lymphocyte recovery and may reduce the risk of graft failure in haploidentical hematopoietic stem-cell transplantation. Blood 110:2764-2767

6. Wakitani S, Imoto K, Yamamoto T, Saito M, Murata N, Yoneda M (2002) Human autologous culture expanded bone marrow mesenchymal cell transplantation for repair of cartilage defects in osteoarthritic knees. Osteoarthritis Cartilage 10:199-206

7. Ross AL, Leder DE, Weiss J, Izakovic J, Grichnik JM (2011) Genomic instability in cultured stem cells: associated risks and underlying mechanisms. Regen Med 6:653-662

8. Miura M, Miura Y, Padilla-Nash HM, Molinolo AA, Fu BJ, Patel V et al (2006) Accumulated chromosomal instability in murine bone marrow mesenchymal stem cells leads to malignant transformation. Stem Cells 24:1095-1103

9. Mohseny AB, Szuhai K, Romeo S, Buddingh EP, Briaire-de Bruijn I, de Jong D et al (2009) Osteosarcoma originates from mesenchymal stem cells in consequence of aneuploidization and genomic loss of Cdkn2. J Pathol 219:294-305

10. Tolar J, Nauta AJ, Osborn MJ, Mortari AP, McElmurry RT, Bell S et al (2007) Sarcoma derived from cultured mesenchymal stem cells. Stem Cells 25:371-379

11. Jeong JO, Han JW, Kim JM, Cho HJ, Park C, Lee N et al (2011) Malignant tumor formation after transplantation of short-term cultured bone marrow mesenchymal stem cells in experimental myocardial infarction and diabetic neuropathy. Circ Res 108:1340-1347

12. Josse C, Schoemans R, Niessen NA, Delgaudine M, Hellin AC, Herens C et al (2010) Systematic chromosomal aberrations found in murine bone marrow-derived mesenchymal stem cells. Stem Cells Dev 19:1167-1173

13. Izadpanah R, Kaushal D, Kriedt C, Tsien F, Patel B, Dufour J et al (2008) Long-term in vitro expansion alters the biology of adult mesenchymal stem cells. Cancer Res 68:4229-4238

14. Bernardo ME, Zaffaroni N, Novara F, Cometa AM, Avanzini MA, Moretta A et al (2007) Human bone marrow-derived mesenchymal stem cells do not undergo transformation after long-term In vitro culture and do not exhibit telomere maintenance mechanisms. Cancer Res 67:9142-9149

15. Rubio D, Garcia-Castro J, Martin MC, de la Fuente R, Cigudosa JC, Lloyd AC et al (2005) Spontaneous human adult stem cell transformation. Cancer Res 65:3035-3039
16. Rubio D, Garcia S, De la Cueva T, Paz MF, Lloyd AC, Bernad A et al (2008) Human mesenchymal stem cell transformation is associated with a mesenchymal-epithelial transition. Exp Cell Res 314:691-698

17. Rubio D, Garcia S, Paz MF, De la CT, Lopez-Fernandez LA, Lloyd AC et al (2008) Molecular characterization of spontaneous mesenchymal stem cell transformation. PLoS One 3:e1398

18. Rosland GV, Svendsen A, Torsvik A, Sobala E, McCormack E, Immervoll $\mathrm{H}$ et al (2009) Long-term cultures of bone marrow-derived human mesenchymal stem cells frequently undergo spontaneous malignant transformation. Cancer Res 69:5331-5339

19. Torsvik A, Rosland GV, Svendsen A, Molven A, Immervoll H, McCormack E et al (2010) Spontaneous malignant transformation of human mesenchymal stem cells reflects cross-contamination: putting the research field on track-letter. Cancer Res 70:6393-6396

20. de la Fuente R, Bernad A, Garcia-Castro J, Martin MC, Cigudosa JC (2010) Retraction: spontaneous human adult stem cell transformation. Cancer Res 70:6682

21. Berger M, Muraro M, Fagioli F, Ferrari S (2008) Osteosarcoma derived from donor stem cells carrying the Norrie's disease gene. N Engl J Med 359:2502-2504

22. Ben-David U, Mayshar Y, Benvenisty N (2011) Large-scale analysis reveals acquisition of lineage-specific chromosomal aberrations in human adult stem cells. Cell Stem Cell 9:97-102

23. Tarte K, Gaillard J, Lataillade JJ, Fouillard L, Becker M, Mossafa H et al (2010) Clinical-grade production of human mesenchymal stromal cells: occurrence of aneuploidy without transformation. Blood 115:1549-1553

24. Fletcher CDM, Bridge JA, Hogendoorn PCW, Mertens F (2013) WHO classification of tumours of soft tissue and bone. International Agency for Research on Cancer, Lyon

25. Szuhai K, Cleton-Jansen AM, Hogendoorn PC, Bovee JV (2012) Molecular pathology and its diagnostic use in bone tumors. Cancer Genet 205:193-204

26. Stephens PJ, Greenman CD, Fu B, Yang F, Bignell GR, Mudie LJ et al (2011) Massive genomic rearrangement acquired in a single catastrophic event during cancer development. Cell 144:27-40

27. Holland AJ, Cleveland DW (2012) Chromoanagenesis and cancer: mechanisms and consequences of localized, complex chromosomal rearrangements. Nat Med 18:1630-1638

28. Fujiwara T, Bandi M, Nitta M, Ivanova EV, Bronson RT, Pellman D (2005) Cytokinesis failure generating tetraploids promotes tumorigenesis in p53-null cells. Nature 437:1043-1047

29. Bernardo ME, Emons JAM, Karperien M, Nauta AJ, Willemze R, Roelofs H et al (2007) Human mesenchymal stem cells derived from bone marrow display a better chondrogenic differentiation compared with other sources. Connect Tissue Res 48:132-140

30. Dominici M, Le Blanc K, Mueller I, Slaper-Cortenbach I, Marini FC, Krause DS et al (2006) Minimal criteria for defining multipotent mesenchymal stromal cells. The International Society for Cellular Therapy position statement. Cytotherapy 8:315-317

31. Hallor KH, Staaf J, Bovee JVMG, Hogendoorn PCW, Cleton-Jansen AM, Knuutila S et al (2009) Genomic profiling of chondrosarcoma: chromosomal patterns in central and peripheral tumors. Clin Cancer Res 15:2685-2694

32. Buddingh EP, Kuijjer ML, Duim RA, Burger H, Agelopoulos K, Myklebost $O$ et al (2011) Tumor-infiltrating macrophages are associated with metastasis suppression in high-grade osteosarcoma: a rationale for treatment with macrophage activating agents. Clin Cancer Res 17:2110-2119

33. Szuhai K, Tanke HJ (2006) COBRA: combined binary ratio labeling of nucleicacid probes for multi-color fluorescence in situ hybridization karyotyping. Nat Protoc 1:264-275

34. Tusher VG, Tibshirani R, Chu G (2001) Significance analysis of microarrays applied to the ionizing radiation response. Proc Natl Acad Sci USA 98:5116-5121

35. Prockop DJ, Keating A (2012) Relearning the lessons of genomic stability of human cells during expansion in culture: implications for clinical research. Stem Cells 30:1051-1052

36. Kim J, Kang JW, Park JH, Choi Y, Choi KS, Park KD et al (2009) Biological characterization of long-term cultured human mesenchymal stem cells. Arch Pharm Res 32:117-126 
37. Pampalona J, Frias C, Genesca A, Tusell L (2012) Progressive telomere dysfunction causes cytokinesis failure and leads to the accumulation of polyploid cells. PLoS Genet 8:e1002679

38. Scielzo C, Ghia P, Conti A, Bachi A, Guida G, Geuna M et al (2005) HS1 protein is differentially expressed in chronic lymphocytic leukemia patient subsets with good or poor prognoses. J Clin Invest 115:1644-1650

39. Frezzato F, Gattazzo C, Martini V, Trimarco V, Teramo A, Carraro S et al (2012) HS1, a Lyn kinase substrate, is abnormally expressed in B-chronic lymphocytic leukemia and correlates with response to fludarabine-based regimen. PLoS One 7:e39902

40. Pansuriya TC, van ER, d'Adamo P, van Ruler MA, Kuijjer ML, Oosting J et al (2011) Somatic mosaic IDH1 and IDH2 mutations are associated with enchondroma and spindle cell hemangioma in Ollier disease and Maffucci syndrome. Nat Genet 43:1256-1261
41. Christensen R, Alsner J, Brandt SF, Dagnaes-Hansen F, Kolvraa S, Serakinc N (2008) Transformation of human mesenchymal stem cells in radiation carcinogenesis: long-term effect of ionizing radiation. Regen Med 3:849-861

42. Ottaviani G, Jaffe N (2009) The etiology of osteosarcoma. Cancer Treat Res 152:15-32

43. Navin N, Kendall J, Troge J, Andrews P, Rodgers L, Mclndoo J et al (2011) Tumour evolution inferred by single-cell sequencing. Nature 472:90-94

44. Zamani EM, Dimitriadou E, Mateiu L, Melotte C, Van der Aa N, Kumar P et al (2015) Concurrent whole-genome haplotyping and copy-number profiling of single cells. Am J Hum Genet 96(6):894-912
Submit your next manuscript to BioMed Central and take full advantage of:

- Convenient online submission

- Thorough peer review

- No space constraints or color figure charges

- Immediate publication on acceptance

- Inclusion in PubMed, CAS, Scopus and Google Scholar

- Research which is freely available for redistribution

Submit your manuscript at www.biomedcentral.com/submit 